\title{
Optimizing Power Efficiency of OFDM Using Quantized Channel State Information
}

\author{
Antonio G. Marqués, Student Member, IEEE, Fadel F. Digham, Member, IEEE, and \\ Georgios B. Giannakis, Fellow, IEEE
}

\begin{abstract}
Emerging applications involving low-cost wireless sensor networks motivate well optimization of orthogonal frequency-division multiplexing (OFDM) in the power-limited regime. To this end, the present paper develops loading algorithms to minimize transmit-power under rate and error probability constraints, using three types of channel state information at the transmitter (CSIT): deterministic (per channel realization) for slow fading links, statistical (channel mean) for fast fading links, and quantized $(Q)$, whereby a limited number of bits are fed back from the transmitter to the receiver. Along with optimal bit and power loading schemes, quantizer designs and reduced complexity alternatives with low feedback overhead are developed to obtain a suite of Q-CSIT-based OFDM transceivers with desirable complexity versus power-consumption tradeoffs. Numerical examples corroborate the analytical claims and reveal that significant power savings result even with a few bits of Q-CSIT.
\end{abstract}

Index Terms-Frequency-selective channels, interpolation techniques, low-power systems, orthogonal frequency-division multiplexing (OFDM), optimization methods, quantization techniques.

\section{INTRODUCTION}

$\mathbf{O}$ RTHOGONAL FREQUENCY-DIVISION MULTIPLEXING (OFDM) has been the "workhorse modulation" for bandwidth-limited wireline and wireless transmissions over frequency-selective multipath channels. Testament to its well-documented merits is provided by the fact that OFDM has been adopted by digital subscriber line (DSL) modems, digital audio and video broadcasting (DAB/DVB) standards, and wireless local area networks, to name a few [12], [19]. Spectral efficiency and error resilience in these applications are well known to improve with the knowledge of channel state information at the transmitter (CSIT). For this reason, OFDM transmissions over wireline or slowly fading wireless links have traditionally relied on deterministic (per channel realization) CSIT to adaptively load power and bits so as to maximize rate (capacity) for a prescribed transmit-power. However, errors in estimating the channel at the receiver, feedback delay, and the asymmetry between forward and reverse links render acquisition of deterministically perfect CSIT pragmatically

Manuscript received September 19, 2005; revised April 15, 2006. The work of A. G. Marqués was supported in part by the Spanish Government under Grant TEC2005-06766-C03-01/TCM. This work was supported in part by the USDoD ARO under Grant W911NF-05-1-0283.

A. G. Marqués is with the Department of Signal Theory and Communications, Rey Juan Carlos University, Cmno. del molino s/n, Fuenlabrada, Madrid 28943, Spain (e-mail: antonio.garcia.marques@urjc.es).

F. F. Digham and G. B. Giannakis are with the Department of Electrical and Computer Engineering, University of Minnesota, Minneapolis, MN 55455 USA (e-mail: fdigham@ece.umn.edu; georgios@ece.umn.edu).

Digital Object Identifier 10.1109/JSAC.2006.879397 impossible in most wireless scenarios. This has motivated OFDM loading schemes based on statistical (S) CSIT or quantized (Q) CSIT, whereby a limited number of bits are fed back from the receiver to the transmitter; see, e.g., [6], [8], [14] and [20], and references therein. But these works too deal with the bandwidth-limited setup, where the objective is to maximize rate or minimize bit-error rate (BER).

Interestingly, except for [17] where deterministic (D) CSIT is used to minimize power consumption, analogous efforts have not been devoted to optimizing OFDM in the power-limited regime. This is the theme of the present paper which aims at loading algorithms to minimize transmit-power under rate and error probability constraints using Q-CSIT. This goal is timely for both commercial, as well as tactical communication systems designed to extend battery lifetime, and especially for wireless networks of sensors equipped with nonrechargeable batteries. Our focus on Q-CSIT is well justified since the resultant transceivers are universally applicable to frequency-selective wireless channels, they incur controllable amount of feedback overhead and implementation complexity, they turn out to be more power-efficient than S-CSIT-based ones, and for a sufficient number of feedback bits they even approach the power savings achieved by the benchmark D-CSIT designs.

The rest of this paper is organized as follows. After introducing preliminaries on the setup we deal with (Section II), we derive optimal power and bit loading schemes for OFDM based on D-CSIT in order to provide the fundamental limit in power efficiency (Section III). Subsequently, we develop the most widely applicable OFDM transceivers based on Q-CSIT which entail two design phases: the offline phase specifying the Q-CSIT format by selecting channel quantization thresholds based on channel mean statistics, and the online one deriving the bit and power loading schemes per OFDM subchannel (Section IV). A suite of practical derivatives are developed next trading-off power savings for low complexity (Section V) and reduced feedback overhead (Section VI). To complete the gamut of pertinent options, power-efficient OFDM schemes based on S-CSIT are also outlined (Section VII). Finally, numerical results and comparisons of the various alternatives are presented (Section VIII), and concluding remarks wrap up this paper (Section IX).

\section{Preliminaries and Problem Statement}

With reference to Fig. 1, we consider wireless OFDM transmissions over $N$ subcarriers (subchannels) through frequencyselective fading channels with discrete-time baseband equivalent impulse response taps $\left\{h_{n}\right\}_{n=0}^{L}$, where $L:=\left\lfloor D_{\max } W\right\rfloor$ denotes the channel order, $D_{\max }$ the maximum delay spread, 


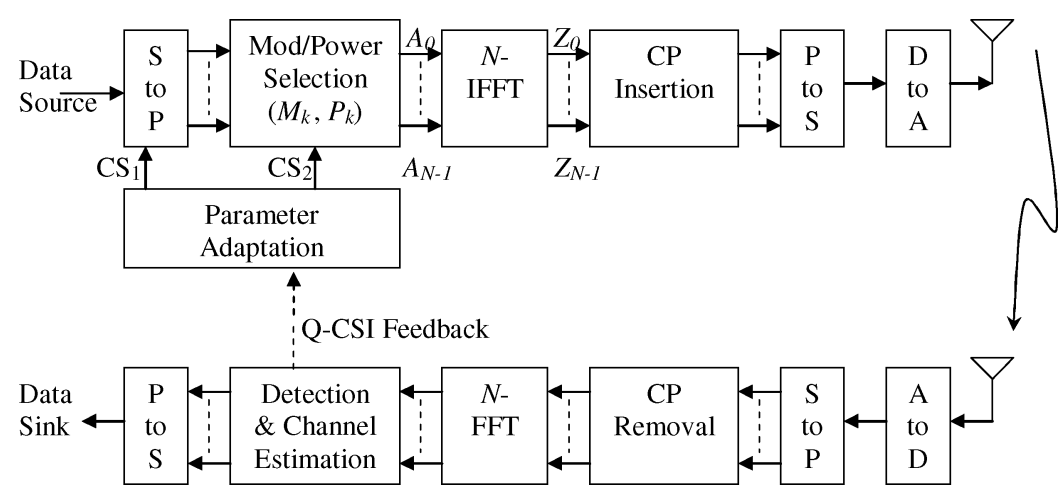

Fig. 1. Transmission system block diagram.

$W$ the transmit-bandwidth, and $\lfloor\cdot\rfloor$ stands for the floor operation. A serial stream of data bits is demultiplexed to form $N$ parallel streams each of which (indexed by $k \in[0, N-1]$ ) is multiplied by an appropriate constant to load power $P_{k}$, and modulated to obtain symbols $\left\{A_{k}\right\}_{k=0}^{N-1}$ drawn from a constellation of size $M_{k}$. The $N$-point inverse fast Fourier transform (I-FFT) applied to each snapshot of the $N$ symbol streams is followed by insertion of a size- $L$ cyclic prefix (CP) to yield a block of $N+L$ symbols (also known as OFDM symbol), which are subsequently multiplexed and digital to analog (D/A) converted for transmission. These operations along with the corresponding FFT and CP removal at the receiver convert the multipath fading frequency-selective channel to a set of $N$ parallel flat-fading subchannels each with fading coefficient given by the FFT (see, e.g., [19]): $H_{k}=(1 / \sqrt{N}) \sum_{n=0}^{N-1} h_{n} e^{-j(2 \pi / N) k n}$, where $N$ is typically chosen so that $N \gg L$.

Channel estimation at the receiver relies on periodically inserted training symbols (also known as pilots). With the channel acquired, the receiver has available a noise-normalized channel gain vector $\mathbf{g}:=\left[g_{0}, \ldots, g_{N-1}\right]^{T}$, where $[.]^{T}$ denotes transposition and $g_{k}:=\left|H_{k}\right|^{2} / \sigma_{k}^{2}$ is the instantaneous noise-normalized channel gain of the $k$ th subchannel on which the zero-mean additive white Gaussian noise (AWGN) has variance $\sigma_{k}^{2}$. Each deterministic realization of the random gain $g_{k}$ constitutes the D-CSI, while its statistical average $\bar{g}_{k}:=\mathbb{E}\left[g_{k}\right]$ is what we henceforth refer to as S-CSI of the $k$ th subchannel $(\mathbb{E}[$.$] denotes$ the expectation operator). Either one must be available to the transmitter in the D-CSIT or S-CSIT-based OFDM designs, but $\bar{g}_{k}$ will also be used in specifying the form of Q-CSIT; i.e., the channel quantizer employed by the Q-CSIT-based OFDM transceivers. The quantizer design entails selection of quantization thresholds $\tau_{k, j}$, which in turn define quantization regions $\mathcal{R}_{k, j}$ each subchannel realization $g_{k}$ falls into. The random vector of bits $\mathbf{j}:=\left[j_{0}, j_{1}, \ldots, j_{N-1}\right]^{T}$ representing indices of the regions of all subchannels is what we term Q-CSI, and is what must be fed back from the receiver to the transmitter in Q-CSIT-based OFDM schemes.

Given either $\mathbf{g}$ (D-CSIT), or its mean $\overline{\mathbf{g}}$ (S-CSIT), or the quantized form of the channel gains specified by the bit vector $\mathbf{j}$ (Q-CSIT), our ultimate goal is to choose power and bit vectors $\mathbf{p}:=\left[P_{0} \ldots P_{N-1}\right]^{T}$ and $\mathbf{m}:=\left[M_{0} \ldots M_{N-1}\right]^{T}$ so as to minimize the power $P:=\sum_{k=0}^{N-1} P_{k}$, or its average $\bar{P}$, for prescribed values of the rate $\left(R_{0}\right)$ and the bit error rate $\left(\mathrm{BER}_{0}\right)$ per OFDM symbol $\mathbf{a}:=\left[A_{0} \ldots A_{N-1}\right]^{T}$. In addition, we wish to choose judiciously the channel quantizer in Q-CSIT-based designs, and develop bit and power loading algorithms which are flexible to achieve desirable tradeoffs among implementation complexity, the required feedback overhead, and power efficiency. We will pursue these objectives under the following assumptions.

a1) Symbols $A_{k}$ are drawn from quadrature amplitude modulation (QAM) constellations of size $M_{k}$.

a2) Subchannels $H_{k}$ remain invariant over at least two consecutive OFDM symbols, they are allowed to be correlated, and each is complex Gaussian distributed; i.e., each subchannel gain $g_{k}$ adheres to an exponential probability density function (pdf) $f_{g_{k}}\left(g_{k}\right)=\left(1 / \bar{g}_{k}\right) \exp \left(-g_{k} / \bar{g}_{k}\right)$.

a3) Feedback channel is error-free and incurs negligible delay.

Assumptions a1-a3 are common to existing designs [6], [8], [12], [14], and are typically satisfied in practice. In principle, our results apply to any channel pdf, but a2 simplifies the resultant designs. Channel invariance in a2 allows for feedback delays; it may be stringent for D-CSIT-based designs, but is very reasonable in the Q-CSIT case since each subchannel can vary from one OFDM symbol to the next so long as the quantization region it falls into remains invariant. Finally, error-free feedback is easily guaranteed with sufficiently strong error control codes especially since rate in the reverse link is low.

With $R$ denoting rate (number of bits per OFDM block), the instantaneous BER is

$$
\mathrm{BER}=\frac{1}{R} \sum_{k=0}^{N-1} \log _{2}\left(M_{k}\right) \mathrm{BER}_{k}\left(g_{k}, P_{k}, M_{k}\right)
$$

where $\operatorname{BER}_{k}\left(g_{k}, P_{k}, M_{k}\right) \simeq a \exp \left(-\beta_{k} g_{k} P_{k}\right)$ and for the QAM constellations in a1, we have $a=0.2, \beta_{k}:=$ $b_{k} /\left(M_{k}-1\right)$, with $b_{k}=1$ if $M_{k}=2$, or, $b_{k}=1.5$ if $M_{k} \geq 4$; and the approximation of the $\mathrm{BER}_{k}$ corresponding to the $k$ th subchannel is very accurate [5], [9]. Notice that for a given $\mathrm{BER}_{k}$, we can express $P_{k}$ as a function of $g_{k}$ and the modulation related constants $a$ and $\beta_{k}$. This expression as well as (1) will come handy in the next section, where we optimize bit and power loading based on D-CSIT. 


\section{OPTIMIZATION BASED ON D-CSIT}

In this section, we rely on the D-CSIT gain vector $\mathrm{g}$ to optimize power efficiency of OFDM for slow fading wireless or wireline channels. Since $\mathrm{g}$ represents the full CSI possibly available, the resultant power and bit allocation also serve as benchmarks for the designs based on partial (namely quantized or statistical) CSI. With $\mathcal{M}$ denoting the finite set of possible values of $\mathbf{m}$, our objective can be formulated as follows:

$$
\begin{aligned}
& \min _{\mathbf{m}(\mathbf{g}) \in \mathcal{M}, \mathbf{p}(\mathbf{g}) \geq \mathbf{0}} J(\mathbf{p}), \quad J(\mathbf{p}):=\sum_{k=0}^{N-1} P_{k}(\mathbf{g}) \\
& \text { s. to } \\
& \text { C1. } \sum_{k=0}^{N-1} \frac{\log _{2} M_{k}}{R_{0}} \operatorname{BER}_{k}\left(g_{k}, P_{k}, M_{k}\right)=\mathrm{BER}_{0} \\
& \text { C2. } \quad \sum_{k=0}^{N-1} \log _{2} M_{k}=R_{0} .
\end{aligned}
$$

Since entries in $\mathbf{p}$ take on continuous values while those in $\mathbf{m}$ are integer-valued, we deal with a mixed-integer programming (MIP) problem, which can be solved in two steps. First, we will solve (2) for a fixed $\mathbf{m}$ (i.e., with $\mathrm{C} 2$ removed and the optimum $\mathbf{p}$ expressed in terms of $\mathbf{m}$ ), and then we will select the overall optimum $(\mathbf{p}, \mathbf{m})$ pair by exhaustively testing over all finite realizations of $\mathbf{m} \in \mathcal{M} \cdot{ }^{1}$ Substituting (1) into (2), the first step is

$$
\begin{aligned}
& \min _{\mathbf{p}(\mathbf{g}) \geq \mathbf{0}} J(\mathbf{p}), \quad J(\mathbf{p})=\sum_{k=0}^{N-1} P_{k}(\mathbf{g}) \\
& \text { s. to } \\
& \text { C1. } \quad \sum_{k=0}^{N-1} \frac{\log _{2} M_{k}}{R_{0}} \exp \left(-\beta_{k} g_{k} P_{k}(\mathbf{g})\right)=\text { BER }_{0} .
\end{aligned}
$$

Invoking the necessary Karush-Kuhn-Tucker (KKT) conditions for optimality [10, Sec. 14.6], the solution $P_{k}(\mathrm{~g})$ can be found to be

$$
P_{k}(\mathbf{g})=\frac{1}{\beta_{k} g_{k}}\left[\ln \left(\frac{a \beta_{k} g_{k} \log _{2} M_{k}}{R_{0}} \lambda_{D}\right)\right]^{+}
$$

where $[x]^{+}:=\max (x, 0)$, and $\lambda_{D}$ is the so-called Lagrange multiplier which can be obtained after plugging (4) into C1. Notice that apart from $\lambda_{D}, P_{k}$ only depends on $g_{k}$, i.e., $P_{k}(\mathrm{~g})=P_{k}\left(g_{k}\right)$. Because both $J(\mathbf{p})$ and $\mathrm{C} 1$ are convex functions of $\mathbf{p}$, the solution in (4) is globally optimum. Reminiscent of the water-filling principle, (4) also shows that depending on subchannel gains, it is possible for certain subchannels to receive zero-power. Let $\mathcal{N}_{a}$ be the set of indices corresponding to the $N_{a}(\leq N)$ active subchannels which are allocated nonzero power. Upon substituting (4) into $\mathrm{C} 1$, we obtain

$$
\lambda_{D}=\frac{1}{\mathrm{BER}_{0}} \sum_{k \in \mathcal{N}_{a}} \frac{1}{\beta_{k} g_{k}}
$$

\footnotetext{
${ }^{1}$ As will be shown shortly in Algorithm 1, the search space over $\mathbf{m}$ can be rendered very confined.
}

We next incorporate the rate constraint $\mathrm{C} 2$ in the second step of our optimization process, and reduce the aforementioned exhaustive search over $\mathbf{m}$ following the algorithm described next.

\section{Algorithm 1}

D1) Sort the subchannels in descending order according to their $g_{k}$ values.

D2) Create a table of all possible $\mathbf{m}$ vectors that satisfy the constraint $\mathrm{C} 2$ ordered according to subchannel gains; i.e., if $g_{k_{1}} \geq g_{k_{2}}$, then $M_{k_{1}} \geq M_{k_{2}}$.

D3) For each $\mathbf{m}$ of the table, evaluate the corresponding $\mathbf{p}$ with entries as in (4), and select the $(\mathbf{m}, \mathbf{p})$ pair that leads to the minimum $J(\mathbf{p})=\sum_{k=0}^{N-1} P_{k}$.

Sorting in D1 confines the search space over $\mathcal{M}$ considerably; see also [17] for a proof. The intuition behind D1 is that the higher $g_{k}$ is, the higher $M_{k}$ can be loaded to minimize $P$.

Similar to the classical water-filling-based loading scheme where rate is maximized subject to a power constraint [3], in our loading scheme for power optimization subject to rate and BER constraints, $M_{k}$ generally increases for larger $g_{k}$ which in turn causes $P_{k}$ to increase. But for subchannels loaded with the same $M_{k}$, power $P_{k}$ decreases as $g_{k}$ increases.

To gain further insight about our solution of (3), let us consider the related problem of minimizing $P$ under a constraint on capacity. In this case, the KKT conditions readily yield the optimum power allocation as

$$
P_{k}(\mathrm{~g})=\left[\zeta_{D}-\frac{1}{g_{k}}\right]^{+}
$$

where $\zeta_{D}=\left(2^{C_{0}} / \prod_{k \in \mathcal{N}_{a}} g_{k}\right)^{1 / N_{a}}$, and $C_{0}$ is the prescribed capacity. It is interesting to notice that absence of the BER constraint here, causes $P_{k}(\mathrm{~g})$ to always increase as $g_{k}$ increases.

Remark 1: Alternative MIP techniques accounting jointly for $\mathrm{C} 1$ and $\mathrm{C} 2$ can in principle be used to solve the optimization problem in (2). However, joint consideration of $\mathrm{C} 1$ and $\mathrm{C} 2$ increases the problem dimensionality and the optimization burden. For instance, applying the branch-and-bound algorithm with Lagrangian relaxation to solve (2) requires solving a set of nonconvex subproblems [1].

Remark 2: In the optimal loading schemes of this section as well as those of the ensuing sections, we did not impose peak-to-average-power-ratio (PAPR) constraints. However, available digital predistortion schemes (such as the selected mapping algorithm in [18]) can be applied to each block of $\left\{A_{k}\right\}_{k=0}^{N-1}$ symbols in order to meet prespecified PAPR constraints.

\section{OPTIMIZATION BASED ON Q-CSIT}

Instead of optimizing power efficiency of OFDM based on the realization $g$ of the subchannel gains, here we rely on the bit vector $\mathbf{j}$ comprising codewords $j_{k}$ which represent the quantization region indices each subchannel gain $g_{k}$ falls into. In this context, we will assume that: 
a4) Codeword $j_{k}$ has length $B \forall k$; hence, $\mathbf{j}$ is an $N B \times 1$ vector with 0,1 entries.

These $B$ bits per subchannel will index $2^{B}$ regions $\mathcal{R}_{k, j}:=$ $\left[\tau_{k, j}, \tau_{k, j+1}\right)$, that the $g_{k}$ space is divided using quantization thresholds $\left[\tau_{k, 1}, \ldots, \tau_{k, 2^{B}-1}\right]^{T}:=\tau_{k}$ for each subchannel $k \in$ $[0, \ldots, N-1]$. Notice that for brevity, we use $j$ (rather than $j_{k}$ ) to index regions and thresholds; however, since the latter include also the subscript $k$, we should keep in mind that regions and thresholds are allowed to be different from one subchannel to another. Further, since quantization here pertains to gains, we set $\tau_{k, 0}=0$ and $\tau_{k, 2^{B}}=\infty$.

Our Q-CSIT-based optimization will proceed in two phases: 1) specification of the optimum thresholds $\left\{\boldsymbol{\tau}_{k}\right\}_{k=0}^{N-1}$, which can be performed offline and 2) derivation of the optimum power and bit loading vectors $(\mathbf{m}, \mathbf{p})$ to be used online based on the Q-CSI codeword vector $\mathbf{j}$ obtained in the quantizer design phase $1)$.

\section{A. Offline Phase: Quantizer Design}

We wish to derive quantization thresholds for the random variable $g_{k}$ which has a known pdf as per a2. Those should clearly depend on the underlying distortion metric which, in par with our objective on power efficiency, will be taken to be the average power $\sum_{k=0}^{N-1} \bar{P}_{k}$. To evaluate $\bar{P}_{k}$, recall that for a prescribed BER the power $P_{k}$ will depend on the quantized $g_{k}$, which takes $2^{B}$ possible values; correspondingly, $P_{k}$ is a discrete random variable taking values $\left\{P_{k, j}\right\}_{j=0}^{2^{B}-1}$ with probability $\operatorname{Pr}\left(P_{k}=P_{k, j}\right)=\operatorname{Pr}\left(g_{k} \in \mathcal{R}_{k, j}\right):=$ $\int_{\tau_{k, j}}^{\tau_{k, j+1}} f_{g_{k}}\left(g_{k}\right) d g_{k}=\exp \left(-\tau_{k, j} / \bar{g}_{k}\right)-\exp \left(-\tau_{k, j+1} / \bar{g}_{k}\right)$, where the last equality comes from the exponential pdf in a2. We can thus readily deduce that $\bar{P}_{k}=\sum_{j=0}^{2^{B}-1} P_{k, j} \operatorname{Pr}\left(P_{k}=P_{k, j}\right)$, which shows how the average power depends on the thresholds and the channel pdf.

Arguing likewise, since $g_{k}$ is a continuous random variable and $P_{k}$ is a discrete one, we can also express the average BER per subchannel as: $\overline{\mathrm{BER}}_{k}\left(\boldsymbol{\tau}_{k},\left\{P_{k, j}\right\}, M_{k}\right)=$ $\sum_{j=0}^{2^{B}-1} \int_{\tau_{k, j}}^{\tau_{k, j+1}} \operatorname{BER}_{k}\left(g_{k}, P_{k, j}, M_{k}\right) f_{g_{k}}\left(g_{k}\right) d g_{k}$. Notice that for the region corresponding to $j=0$, we have $P_{k, 0}=\mathrm{BER}_{k}=$ 0 . For the quantization thresholds to be suitable across channel realizations, we will use the average power as objective function and include the average BER in our constraints. Thus, our channel quantizer design for selecting thresholds can be formulated as follows:

$$
\begin{aligned}
& \min _{\tau_{k, j}(B, \overline{\mathbf{g}}) \geq 0} J(\overline{\mathbf{p}}), \quad J(\overline{\mathbf{p}}):=\sum_{k=0}^{N-1} \bar{P}_{k}(B, \overline{\mathbf{g}}) \\
& \text { s. to } \\
& \text { C1. } \sum_{k=0}^{N-1} \frac{\log _{2} M_{k}}{R_{0}} \overline{\mathrm{BER}}_{k}\left(\boldsymbol{\tau}_{k},\left\{P_{k, j}\right\}, M_{k}\right)=\mathrm{BER}_{0} \\
& \text { C2. } \quad \sum_{k=0}^{N-1} \log _{2} M_{k}=R_{0} .
\end{aligned}
$$

As in our D-CSIT-based optimization, we will first solve (7) without accounting for $\mathrm{C} 2$. Upon substituting the expressions for $\bar{P}_{k}$ and $\overline{\mathrm{BER}}_{k}$ into (7) and defining $\gamma_{k, j}:=\beta_{k} P_{k, j}+1 / \bar{g}_{k}$, the objective function $J(\overline{\mathbf{p}})$ and constraint $\mathrm{C} 1$ for this relaxed problem can be written, respectively, as

$$
\begin{aligned}
& \sum_{k=0}^{N-1} \sum_{j=1}^{2^{B}-1} P_{k, j}\left(e^{-\frac{\tau_{k, j}}{\bar{g}_{k}}}-e^{-\frac{\tau_{k, j+1}}{\bar{g}_{k}}}\right) . \\
& \sum_{k=0}^{N-1} \frac{a \log _{2} M_{k}}{R_{0} \bar{g}_{k}} \sum_{j=1}^{2^{B}-1} \frac{e^{-\gamma_{k, j} \tau_{k, j}}-e^{-\gamma_{k, j} \tau_{k, j+1}}}{\gamma_{k, j}}=\text { BER }_{0} .
\end{aligned}
$$

Lack of convexity that we demonstrate in Appendix A implies that only locally optimal solutions can be ensured in our quantizer design. Nonetheless, the Lagrangian for minimizing (8) subject to (9) is given by

$$
\begin{aligned}
\mathcal{L} & =\ldots+P_{k, j-1} \int_{\tau_{k, j-1}}^{\tau_{k, j}} f_{g_{k}}\left(g_{k}\right) d g_{k}+P_{k, j} \int_{\tau_{k, j}}^{\tau_{k, j+1}} f_{g_{k}}\left(g_{k}\right) d g_{k} \\
& +\ldots \\
& +\lambda_{Q}\left\{\sum _ { k } \frac { a \operatorname { l o g } _ { 2 } M _ { k } } { R _ { 0 } } \left[\ldots+\int_{\tau_{k, j-1}}^{\tau_{k, j}} e^{-\beta_{k} g_{k} P_{k, j-1}} f_{g_{k}}\left(g_{k}\right) d g_{k}\right.\right. \\
& \left.\left.+\int_{\tau_{k, j}}^{\tau_{k, j+1}} e^{-\beta_{k} g_{k} P_{k, j}} f_{g_{k}}\left(g_{k}\right) d g_{k}+\ldots\right]-\mathrm{BER}_{0}\right\}
\end{aligned}
$$

where $\lambda_{Q}$ is found after fulfilling (9). Upon defining $\psi\left(\gamma_{k, j}, \tau_{k, j}\right):=\left(1+\gamma_{k, j} \tau_{k, j}\right) e^{-\gamma_{k, j} \tau_{k, j}}$, the necessary conditions $\partial \mathcal{L} / \partial \tau_{k, j}=0$ and $\partial \mathcal{L} / \partial P_{k, j}=0$, respectively, yield (for $k=0,1, \ldots N-1$ and $j=1, \ldots 2^{B}-1$ )

$$
\begin{gathered}
P_{k, j-1}-P_{k, j}+\lambda_{Q} \frac{a}{R_{0}} \log _{2}\left(M_{k}\right) \\
\times\left[e^{-\beta_{k} \tau_{k, j} P_{k, j-1}}-e^{-\beta_{k} \tau_{k, j} P_{k, j}}\right]=0 \\
e^{-\frac{\tau_{k, j}}{\bar{g}_{k}}}-e^{-\frac{\tau_{k, j+1}}{\bar{g}_{k}}}-\frac{\lambda_{Q} a \beta_{k} \log _{2} M_{k}}{R_{0} \bar{g}_{k} \gamma_{k j}^{2}} \\
\times\left[\psi\left(\gamma_{k, j}, \tau_{k, j}\right)-\psi\left(\gamma_{k, j}, \tau_{k, j+1}\right)\right]=0 .
\end{gathered}
$$

Equations (11) and (12) can be compactly written as nonlinear functions of the powers and thresholds as $\mathcal{Z}_{1}\left(P_{k, j-1}, P_{k, j}, \tau_{k, j}\right)=0$ and $\mathcal{Z}_{2}\left(P_{k, j}, \tau_{k, j}, \tau_{k, j+1}\right)=0$, respectively. We solve them to obtain $\tau_{k, j}$ using the following offline algorithm.

\section{Algorithm 2}

Q1) Sort the subchannels in decreasing order based on $\bar{g}_{k}$ and generate a table of possible $\mathbf{m}$ candidates satisfying the rate constraint as in steps D1 and D2 of Algorithm

1. Then, execute the following loop for each entry of $\mathbf{m}$.

Q2) For $\lambda_{Q}$ values:

- For $k=0: N-1$

- For $\tau_{k, 1}=0: \tau_{\max }$, where $\tau_{\max }$ is found so that e.g., $\operatorname{Pr}\left(g_{k}>\tau_{\max }\right)=0.01$;

Given a $\tau_{k, 1}$ value, solve (11) numerically (e.g., using the bisection method [15]) and obtain a candidate root $P_{k, 1}\left(\right.$ recall $\left.P_{k, 0}=0\right)$. 
With $P_{k, 1}$ given, solve (12) to determine $\tau_{k, 2}$. Similarly, keep alternating between (11) and (12) to eventually obtain $\left\{P_{k, j}\right\}$ and $\left\{\tau_{k, j}\right\}$ for all $j=1, \ldots, 2^{B}-2$. As these power and threshold values per subchannel are found without using (12) with $j=2^{B}-1$, this equation can be used to test the feasibility of the candidate solution obtained.

- End $\tau_{k, 1}$ loop.

- End $k$ loop.

- At this point, we have a list of candidate solutions $\boldsymbol{\tau}_{k}, \mathbf{p}$. It remains to filter these solutions and accept only those achieving the average BER constraint in (9).

End $\lambda_{Q}$ loop.

Q3) Among all feasible $\boldsymbol{\tau}$ 's, choose the one leading to the minimum $J(\overline{\mathbf{p}})$ in $(8)$.

In step Q1, each choice of $\left\{M_{k}\right\}_{k=1}^{N_{a}}$ satisfies: 1) $N_{a} \leq N$ and 2) $M_{k_{1}} \geq M_{k_{2}}$ if $\bar{g}_{k_{1}} \geq \bar{g}_{k_{2}}$. This means that Algorithm 2 yields the thresholds only for the $N_{a}$ subchannels that are "best on the average". Since on an OFDM symbol-by-symbol basis, we may need thresholds for all subchannels, numerical simulations have suggested a rule of thumb whereby for each $j$, the ratio $\tau_{k, j} / \bar{g}_{k}$ remains invariant for all $k$. This rule will lead naturally to one of the schemes we introduce in the next section.

In step Q2, given an initial $\lambda_{Q}$ value, different algorithms can be used to update $\lambda_{Q}$ [1, Ch. 4]. For example, using the method of multipliers entails $\lambda_{Q}^{(i+1)}=\lambda_{Q}^{(i)}+$ $c^{(i)}\left[\sum_{k=0}^{N-1}\left(\log _{2} M_{k} / R_{0}\right) \overline{\mathrm{BER}}_{k}\left(\boldsymbol{\tau}_{k},\left\{P_{k, j}\right\}, M_{k}\right)-\mathrm{BER}_{0}\right]$, where $i$ denotes the iteration index and $c$ is the penalty parameter that can be updated using different ad hoc methods.

Remark 3: In addition to thresholds, Algorithm 2 yields as a by-product $(\mathbf{m}, \mathbf{p})$ loadings minimizing average power subject to average BER based on statistical CSI (here, the average gains $\left.\bar{g}_{k}\right)$. This $(\mathbf{m}, \mathbf{p})$ pair can be used in simpler but suboptimum loading algorithms we will explore in Section V-C. But first, we will see next how instead of $\bar{g}_{k}$, the optimum online algorithm based on the Q-CSIT codeword $\mathbf{j}$ can yield improved $(\mathbf{m}, \mathbf{p})$ loadings given the quantization thresholds.

\section{B. Online Phase: Power and Bit Loading}

With the quantizer parameters (thresholds and thus regions) fixed, each gain realization $\mathrm{g}$ is estimated and quantized at the receiver to obtain the codeword $\mathbf{j}$. Feeding back this form of instantaneous Q-CSIT, the transmitter will find online the pair of loadings $(\mathbf{m}, \mathbf{p})$ which minimize power subject to rate, and (what we could call conditioned on $\mathbf{j}$ ) average BER per subchannel. With $\gamma_{k}:=\beta_{k} P_{k}+1 / \bar{g}_{k}$, the latter can be expressed as

$$
\begin{aligned}
\overline{\operatorname{BER}}_{k \mid \mathbf{j}} & =\frac{\int_{\tau_{k, j}}^{\tau_{k, j+1}} \operatorname{BER}_{k}\left(P_{k}, g_{k}\right) f_{g_{k}}\left(g_{k}\right) d g_{k}}{\operatorname{Pr}\left(g_{k} \in \mathcal{R}_{k, j}\right)} \\
& =\frac{a\left(e^{-\gamma_{k} \tau_{k, j}}-e^{-\gamma_{k} \tau_{k, j+1}}\right)}{\bar{g}_{k} \gamma_{k}\left(e^{-\tau_{k, j} / \bar{g}_{k}}-e^{-\tau_{k, j+1} / \bar{g}_{k}}\right)} .
\end{aligned}
$$

With this conditional average BER, our optimization problem can be formulated as follows:

$$
\min _{\mathbf{m}(\mathbf{j}) \in \mathcal{M}, \mathbf{p}(\mathbf{j}) \geq \mathbf{0}} \quad J(\mathbf{p}), \quad J(\mathbf{p}):=\sum_{k=0}^{N-1} P_{k}(\mathbf{j})
$$

s. to

$$
\begin{array}{ll}
\text { C1. } & \sum_{k=0}^{N-1} \frac{\log _{2} M_{k}}{R_{0}} \overline{\mathrm{BER}}_{k \mid \mathbf{j}}\left(\tau_{k, j}, \tau_{k, j+1}, P_{k}, M_{k} \mid \mathbf{j}\right)=\mathrm{BER}_{0} \\
\text { C2. } & \sum_{k=0}^{N-1} \log _{2} M_{k}=R_{0} .
\end{array}
$$

Comparing (14) with (7), we infer that apart from the fact that the quantizer design and the regions are given, the structure of both problems is similar. Since the scalar in the denominator of (13) will just alter the $\lambda_{Q}$ multiplier, a simplified version of Algorithm 2 which does not search over thresholds and does not account for (11) can be applied to solve (14) as well. Furthermore, because here we wish to solve for the $(\mathbf{m}, \mathbf{p})$ pair as in $\mathrm{Al}$ gorithm 1 (rather than the thresholds we sought in Algorithm 2), we expect convexity to ensure global optimality. Indeed, the sufficient conditions for optimality require $\partial^{2} J(\mathbf{p}) / \partial P_{k}^{2} \geq 0$, and $\partial^{2} \overline{\mathrm{BER}}_{\mathbf{j}} / \partial P_{k}^{2} \geq 0$. The first condition can be readily verified, while the latter yields

$$
\begin{aligned}
\frac{\partial^{2} \overline{\mathrm{BER}}_{\mid \mathbf{j}}}{\partial P_{k}^{2}}= & \frac{a \log _{2} M_{k}}{R_{0}\left(e^{-\tau_{k, j} / \bar{g}_{k}}-e^{\left.-\tau_{k, j+1} / \bar{g}_{k}\right)}\right.} \\
& \times \int_{\tau_{k, j}}^{\tau_{k, j+1}}\left(\beta_{k} g_{k}\right)^{2} e^{-\gamma_{k} g_{k}} d g_{k} \geq 0
\end{aligned}
$$

where the last inequality follows since $\tau_{k, j}<\tau_{k, j+1}$ and the integrand is non-negative.

\section{REDUCED COMPLEXITY OPTIMIZATION BASED ON Q-CSIT}

Per iteration of the quantizer design in Algorithm 2 (corresponding to each feasible $\mathbf{m}$ ), we look for $2^{B}-1$ thresholds $\tau_{k, j}$, and $2^{B}-1$ power levels $P_{k, j}$ per subchannel. Repeated for all feasible $\mathbf{m}$, this requires searching over $2 N\left(2^{B}-1\right)$ unknowns. Likewise, the online search space entails $N\left(2^{B}-1\right)$ unknowns to solve for $P_{k, j}$ in all subchannels for each $\mathbf{m}$. As $N$ and $B$ increase, this motivates well the reduced complexity, albeit suboptimal, schemes we develop in this section.

\section{A. Reduced Complexity Optimization and Loading}

The key to eliminating half of the unknowns in both phases of the optimization process is to express power levels $P_{k, j}$ in terms of the thresholds $\tau_{k, j}$. To do so, our approach here is to capitalize on the optimum $P_{k}\left(g_{k}\right)$ [c.f., (4)] obtained for the D-CSIT case and examine four reduced complexity (RC) alternatives.

RC1) Estimate the quantized channel gain as $\hat{g}_{k, j}=\left(\tau_{k, j}+\right.$ $\left.\tau_{k, j+1}\right) / 2$, and use (4) to obtain $P_{k, j}=P_{k}\left(\hat{g}_{k, j}\right)$. 
RC2) Utilize directly the pdf in a2 to estimate channel gains via

$$
\begin{aligned}
\hat{g}_{k, j}= & \mathbb{E}\left[\hat{g}_{k} \mid j\right]=\mathbb{E}\left[\hat{g}_{k} \mid \tau_{k, j}<g_{k}<\tau_{k, j+1}\right] \\
= & \frac{1}{e^{-\gamma_{k, j} / \bar{g}_{k}}-e^{-\gamma_{k, j+1} / \bar{g}_{k}}} \\
& \quad \times\left[\tau_{k, j} e^{-\tau_{k, j} / \bar{g}_{k}}-\tau_{k, j+1} e^{-\tau_{k, j+1} / \bar{g}_{k}} \bar{g}_{k}\right. \\
& \left.\quad \times\left(e^{-\tau_{k, j} / \bar{g}_{k}}-e^{-\tau_{k, j+1} / \bar{g}_{k}}\right)\right]
\end{aligned}
$$

and then employ (4) again to obtain power levels as $P_{k, j}=P_{k}\left(\hat{g}_{k, j}\right)$.

RC3) Bypass estimation of the quantized $g_{k}$, by using directly the $\tau_{k, j}$-dependent power quantizer (see also [4])

$$
P_{k, j}=\left.\frac{1}{2} P_{k}\left(g_{k}\right)\right|_{g_{k}=\tau_{k, j}}+\left.\frac{1}{2} P_{k}\left(g_{k}\right)\right|_{g_{k}=\tau_{k, j+1}} .
$$

RC4) Rely as in RC2 on the channel pdf, but use instead the more precise power quantizer $P_{k, j}=\mathbb{E}\left[P_{k}\left(g_{k}\right) \mid \tau_{k, j}<\right.$ $\left.g_{k}<\tau_{k, j+1}\right]$, which can be evaluated using (4) and numerical integration.

Among these four options, RC1 (middle point of the gain quantization region) is the simplest, but as simulations confirm it also leads to the least power efficient Algorithm 2. On the other hand, $\mathrm{RC} 4$ (expected power) is slightly better than the RC2 and RC3 but is rather complex as it does not lead to a closed-form expression of $P_{k, j}$ in terms of $\tau_{k, j}$. Eventually, RC3 (power middle point) is the most promising when considering both complexity and power efficiency. For this reason, we henceforth use (17) that can be rewritten using (4) as

$$
\begin{aligned}
& P_{k, j}=\frac{1}{2 \beta_{k}}\left\{\frac{\left[\ln \left(\beta_{k} \tau_{k, j} \log _{2}\left(M_{k}\right) \lambda_{1}\right)\right]^{+}}{\tau_{k, j}}\right. \\
& \left.+\frac{\left[\ln \left(\beta_{k} \tau_{k, j+1} \log _{2}\left(M_{k}\right) \lambda_{1}\right)\right]^{+}}{\tau_{k, j+1}}\right\}
\end{aligned}
$$

where $\lambda_{1}$ is a constant (that we will term power quantization parameter), which replaces $\lambda_{D}$, and as we will see later has to be determined jointly with the Lagrange multiplier $\lambda_{Q}$ corresponding to the Q-CSIT-based algorithm. Based on (18), we will simplify the offline phase (by optimizing only over $\tau_{k, j}$ ), as well as the online phase (by reducing optimization to a one-dimensional search over $\lambda_{1}$ ).

\section{B. Reduced Complexity Offline Phase}

We develop in this subsection two suboptimum yet computationally efficient schemes that will reduce complexity in the quantizer design phase.

1) Common Thresholds (CT) for All Subchannels: Here, we drop the threshold subscript $k$ and use $\tau_{k, j}=\tau_{j}$ for all $k$, which leads to an $N$-fold reduction in the threshold search space (only $2^{B}-1$ unknowns are involved in each iteration of Algorithm 2). Straightforward application of the KKT conditions with carried out simplifications yields the optimum $2^{B}-1 \mathrm{CT}$ as the solution of the following equations (for $j=1, \ldots, 2^{B}-1$ ):

$$
\begin{aligned}
& \sum_{k=0}^{N-1} \rho_{k, j}^{(j)}\left(e^{-\tau_{j} / \bar{g}_{k}}-e^{-\tau_{j+1} / \bar{g}_{k}}\right)-\frac{P_{k, j}}{\bar{g}_{k}} e^{-\tau_{j} / \bar{g}_{k}} \\
& +\rho_{k, j-1}^{(j)}\left(e^{-\tau_{j-1} / \bar{g}_{k}}-e^{-\tau_{j} / \bar{g}_{k}}\right)+\frac{P_{k, j-1}}{\bar{g}_{k}} e^{-\tau_{j} / \bar{g}_{k}} \\
& \quad-\frac{\lambda_{C T}}{R_{0}} \sum_{k=0}^{N-1} \frac{a \log _{2} M_{k}}{\bar{g}_{k}} \\
& \times\left\{\frac{\rho_{k, j}^{(j)} \beta_{k}\left(e^{\gamma_{k, j} \tau_{j}}-e^{\gamma_{k, j} \tau_{j+1}}\right)}{\gamma_{k, j}^{2}}\right. \\
& \quad+\frac{\left(\gamma_{k, j}-\tau_{j} \beta_{k} \rho_{k, j}^{(j)}\right) e^{\gamma_{k, j} \tau_{j}}+\tau_{j+1} \beta_{k} \rho_{k, j}^{(j)} e^{\gamma_{k, j} \tau_{j+1}}}{\gamma_{k, j}} \\
& \quad+\frac{\rho_{k, j-1}^{(j)} \beta_{k}\left(e^{\gamma_{k, j-1} \tau_{j-1}}-e^{\gamma_{k, j-1} \tau_{j}}\right)}{\gamma_{k, j-1}^{2}} \\
& \quad-\frac{\left(\gamma_{k, j-1}-\tau_{j} \beta_{k} \rho_{k, j-1}^{(j)}\right) e^{\gamma_{k, j-1} \tau_{j}}}{\gamma_{k, j-1}} \\
& \left.+\frac{\tau_{j-1} \beta_{k} \rho_{k, j-1}^{(j)} e^{\gamma_{k, j-1} \tau_{j-1}}}{\gamma_{k, j-1}}\right\}=0
\end{aligned}
$$

where $\lambda_{C T}$ is the Lagrange multiplier and $\rho_{k, i}^{(j)} \triangleq\left(\partial P_{k, i} / \partial \tau_{j}\right)=$ $\left[1-\ln \left(\beta_{k} \tau_{j} \log _{2}\left(M_{k}\right) \lambda_{1}\right)\right] /\left(2 \beta_{k} \tau_{j}^{2}\right)$ if $i=j$ or $i=j-1$ and $\lambda_{1}>1 /\left[\beta_{k} \log _{2}\left(M_{k}\right) \tau_{k j}\right]$, and zero otherwise.

Iterations then proceed as in Algorithm 2 with the exception that we need two loops to search for the Lagrange multiplier $\lambda_{\mathrm{CT}}$ and the power quantization parameter $\lambda_{1}$ (involved in $P_{k, j}$ and $\rho_{k, i}^{(j)}$ ). Upon solving for the thresholds and $\lambda_{1}, P_{k, j}$ can be obtained using (18). Even though this CT-QCSIT is simple, it may not effectively exploit the statistics of the subchannel gains. In the next subsection, we derive instead a simpler yet more efficient scheme to solve for the thresholds.

2) Subcarrier Irrespective Thresholds (SIT): Here, we rely on the rule of thumb we mentioned before Remark 3, which asserts that for each $j, \tau_{k, j} \simeq c_{j} \bar{g}_{k}$ for some constant $c_{j}$ irrespective of $k$. Notice that unlike CT, this SIT-based design accounts for the individual subchannel means, and depending on the distortion metric adopted, we can devise two simplified quantizers: one enforcing equiprobable (EP) quantization regions, and another minimizing the mean-square error in quantizing either the gains (what we naturally term Lloyd- $g_{k}$ quantizer), or, the powers (what we call Lloyd- $P_{k}$ quantizer).

a) EP quantization: In this case, we determine $\tau_{k, j}$ 's so that $\operatorname{Pr}\left(g_{k} \in \mathcal{R}_{k, j}\right)=\int_{\tau_{k, j}}^{\tau_{k, j+1}} f_{g_{k}}\left(g_{k}\right) d g_{k}=1 / 2^{B} \forall k, j$. Under the pdf in a2, this yields the following closed-form solution:

$$
\tau_{k, j}=\bar{g}_{k} \ln \left(\frac{2^{B}}{2^{B}-j}\right)
$$

which interestingly adheres to the aforementioned rule that $\tau_{k, j} \simeq c_{j} \bar{g}_{k}$, and asserts a means of optimality to the EP quantizer. Notice that the closed-form expression (20) reduces 
TABLE I

Number of Iterations ReQuiRed For the DifFERENT METHods PRoPosed

\begin{tabular}{|l|l|l|c|c|}
\hline Phase & Section & Method & Number of iterations w/o RCx & Number of iterations w/ RCx \\
\hline \multirow{4}{*}{ Off-line } & IV.A & Alg. 2 (off) & $2^{B} N_{a} N_{M} I_{\lambda} N_{\tau}\left(I_{P}+I_{\tau}\right)$ & - \\
\cline { 2 - 5 } & V.B.1 & CT & $2^{B} N_{a} N_{M} I_{\lambda} N_{\tau}\left(I_{P}+I_{\tau} / N_{a}\right)$ & $2^{B} N_{M} I_{\lambda} N_{\tau} I_{\tau}$ \\
\cline { 2 - 5 } & V.B.2.a & SIT-EP & {$\left[2^{B} N_{a}, 2^{B} N_{a}\left(N_{M} I_{\lambda} I_{P}+1\right)\right]$} & {$\left[2^{B} N_{a}, 2^{B} N_{a}\left(N_{M} I_{\lambda}+1\right)\right]$} \\
\cline { 2 - 5 } & V.B.2.b & SIT-Lloyd & {$\left[2^{B} N_{a} I_{L}, 2^{B} N_{a}\left(N_{M} I_{\lambda} I_{P}+I_{L}\right)\right]$} & {$\left[2^{B} N_{a} N_{L l}, 2^{B} N_{a}\left(N_{M} I_{\lambda}+I_{L}\right)\right]$} \\
\hline \multirow{5}{*}{ On-line } & IV.B & Alg. 2 (on) & $N_{a} N_{M} I_{\lambda} I_{P}$ & - \\
\cline { 2 - 5 } & V.C & Tx A & $N_{a} N_{M} I_{\lambda} I_{P}$ & $N_{a} N_{M} I_{\lambda}$ \\
\cline { 2 - 5 } & V.C & Tx B & $N_{a} I_{\lambda} I_{P}$ & $N_{a} I_{\lambda}$ \\
\cline { 2 - 5 } & V.C & Tx C & $N_{a} I_{P}$ & $N_{a}$ \\
\cline { 2 - 5 } & V.C & Tx D & $I_{\lambda} I_{P}$ & $I_{\lambda}$ \\
\hline
\end{tabular}

complexity also in the online phase since the average BER constraint in (14) becomes

$$
\begin{aligned}
\overline{\mathrm{BER}}_{E P \mid \mathbf{j}} & =\frac{2^{B} a}{R_{0}} \sum_{k=0}^{N-1} \frac{\log _{2} M_{k}}{\bar{g}_{k} \gamma_{k, j}} \\
& \times\left[\left(\frac{2^{B}-j}{2^{B}}\right)^{\bar{g}_{k} \gamma_{k}}-\left(\frac{2^{B}-j-1}{2^{B}}\right)^{\bar{g}_{k} \gamma_{k}}\right] .
\end{aligned}
$$

b) Lloyd scalar quantization: In this case, we adopt the scalar Lloyd algorithm [7], [13], which for a random variable $x$ with pdf $f_{x}(x)$ quantized to $\hat{x}$ (using $2^{B}$ levels) is known to minimize $\mathbb{E}\left[(x-\hat{x})^{2}\right]$. The quantized $\hat{x}$ and thresholds $\tau_{j}$, are obtained by iteratively solving in an alternating fashion the equations: $\tau_{j}=(1 / 2)\left(\hat{x}_{j}+\hat{x}_{j+1}\right)$ and $\hat{x}_{j}=\left(\int_{\tau_{j}}^{\tau_{j+1}} x f_{x}(x) d x / \int_{\tau_{j}}^{\tau_{j+1}} f_{x}(x) d x\right)$. We can apply Lloyd's algorithm to our problem with either $x=g_{k}$ or $x=P_{k}$ (what we referred to as Lloyd- $g_{k}$ or Lloyd- $P_{k}$ quantizers).

Remark 4: The SIT schemes designed during the offline phase are employed by the optimum online algorithm that uses (18) to obtain $(\mathbf{m}, \mathbf{p})$. Although SIT treats subchannels separately, the online algorithm accounts for subchannels jointly through the conditional average BER constraint.

\section{Reduced Complexity Online Phase}

As we mentioned in Section V-A, (18) can be used in the online phase to simplify the search required to satisfy $\mathrm{C} 1$ in (14) for each feasible m. In Algorithm 2, for each value of $\lambda_{Q}$, we perform $N_{a}$ searches using (12) to determine the power allocation and second check $\mathrm{C} 1$ with the current Lagrange multiplier, repeating this process until $\mathrm{C} 1$ is satisfied. However, using (18), we only need one single search over $\lambda_{1}$ to satisfy $\mathrm{C} 1$, thus reducing complexity drastically.

Furthermore, as we mentioned in Remark 3, Algorithm 2 yields as a by-product a pair of suboptimum loadings $(\mathbf{m}, \mathbf{p})$ in the offline phase. These can be used to reduce complexity also during the online phase by having the transmitter operate in any of the following modes (sorted in decreasing power efficiency but also in decreasing complexity order).

TxA) Select optimally the set of active subcarriers $\mathcal{N}_{a}$, bit loading $\mathbf{m}$, and the value of the Lagrange multiplier, $\lambda$, to attain the global optimum $\mathbf{p}$.

TxB) Select optimally $\mathcal{N}_{a}$ and $\lambda$ while using a fixed $\mathbf{m}$ (no constellation adaptation).
TxC) Select optimally $\mathcal{N}_{a}$, but use fixed values for $\mathbf{m}$ and $\lambda$.

TxD) Select optimally $\lambda$, but use always the same $\mathcal{N}_{a}$ and m.

It is then up to the designer to select among TxA-TxD depending on application specific constraints.

\section{Complexity Comparison}

Let $N_{M}$ denote the number of possible $\mathbf{m}$ candidates which satisfy the rate constraint, $N_{\tau}$ the number of candidate values for the first threshold in each region, $I_{\lambda}$ the number of iterations needed to solve for the Lagrange multiplier associated with the BER constraint, $I_{P}$ the number of iterations needed to determine each power level using any root-finding method (given the values of other parameters), $I_{\tau}$ the number of iterations required to obtain one threshold solution and $I_{L}$ the number of iterations needed until the Lloyd algorithm converges [c.f. Section V-B2b]. Table I summarizes the computational complexity for the various design methods contrasting online and offline phases and illuminating the potential reduction when any of the RC alternatives is employed. As shown, using CT with an RCx alternative (specifically with RC3 as analyzed in Section V-B1) reduces complexity considerably. For the SIT case, the thresholds can be obtained directly or in conjunction with $(\mathbf{m}, \mathbf{p})$. Correspondingly, the notation $[x, y]$ introduced in Table I reduces to $x$ when we are only interested in the quantizer design and reduces to $y$ when $(\mathbf{m}, \mathbf{p})$ are also evaluated on average to be used in the online phase by Tx-B, Tx-C, or Tx-D. More importantly, Table I reveals that the online complexity can be as simple as selecting the active subcarriers or just solving for one unknown, namely, $\lambda$.

So far, we have been using $B$ bits of feedback per subchannel for a total of $N B$ feedback bits, which may be prohibitive as $N$ increases and the channel coherence time decreases. To handle such cases, we will exploit the statistical dependence among subchannels (especially when $N \gg L$ ) and introduce in the ensuing section an efficient means of reducing the feedback overhead.

\section{Q-CSIT WITH REDUCED FEEDBACK OVERHEAD}

It can be readily verified that $L+1$ points on the discrete Fourier transform (DFT) grid suffice to identify the $L+1$ nonzero $h_{n}$ taps. Based on this fact, we prove in Appendix B the following.

Proposition 1: Only $(2 L+1) B$ bits suffice to quantize the channel gain vector $\mathrm{g}$. 


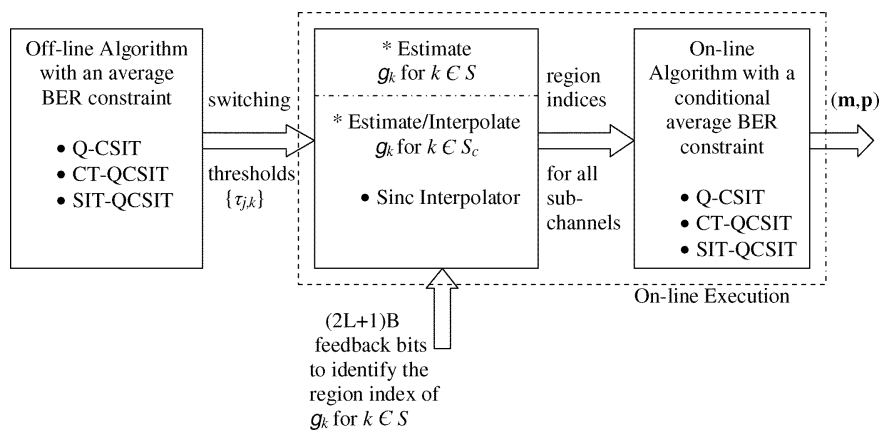

Fig. 2. Interdependence computation modules.

For $N \gg L$, this reduces the required number of feedback bits considerably: from $N B$ to $(2 L+1) B$. Because $2 L+1$ samples of $g_{k}$ on the DFT grid suffice to fully identify the entire gain profile, we let $S$ denote the set of the indices of the $2 L+1$ uniformly sampled subchannels and $S_{c}$ its complement. Given $j_{k}$, we first form the estimates $\hat{g}_{k}$ for $k \in S$, and then interpolate to obtain the remaining $g_{k}$ 's for $k \in S_{c}$. Notice that any of the offline schemes described in the preceding section can be used first to solve for the thresholds in all subchannels. Then, the two aforementioned estimation/interpolation tasks are executed at the transmitter during the online phase. Afterwards, any of the online algorithms discussed in the previous section can be implemented. Fig. 2 depicts the different computation modules along with their interactions. The first task of the estimation/interpolation module, namely subchannel estimation, can be accomplished by using any of the four RC options discussed in the previous section. In RC3 and RC4, we estimate power which is then used to estimate $g_{k}$ for $k \in S$. Taking RC3 as an example and having available the thresholds as well as the offline (while evaluating thresholds) values of the parameters $\beta_{k}$ and $\lambda_{1}, \hat{g}_{k}$ can be found via (18) by solving

$$
\begin{array}{r}
\frac{\left[\ln \left(\beta_{k} \hat{g}_{k} \log _{2}\left(M_{k}\right) \lambda_{1}\right)\right]^{+}}{\hat{g}_{k}}=\frac{\left[\ln \left(\beta_{k} \tau_{k, j} \log _{2}\left(M_{k}\right) \lambda_{1}\right)\right]^{+}}{2 \tau_{k, j}} \\
+\frac{\left[\ln \left(\beta_{k} \tau_{k, j+1} \log _{2}\left(M_{k}\right) \lambda_{1}\right)\right]^{+}}{2 \tau_{k, j+1}}, \quad k \in S .
\end{array}
$$

These different methods of estimation will be compared in Section VIII.

Given $\hat{g}_{k}$ for $k \in S$, our next task is to interpolate and estimate $g_{k}$ for $k \in S_{c}$. Various interpolators are possible but our numerical results suggest that the sinc interpolator leads to the most power efficient scheme. Upon defining $S:=\{\lfloor N /(4 L+$ $1)+n N /(2 L+1)\rfloor \mid n=0, \ldots, 2 L\}$, the sinc interpolator in our case yields

$$
\hat{g}_{k}=\sum_{k^{\prime} \in S} \hat{g}_{k^{\prime}} \operatorname{sinc} \frac{\left(k-k^{\prime}\right)(2 L+1)}{N}, \quad k \in S_{c} .
$$

Up to this point, we explored D-CSIT and Q-CSIT-based designs. To complement our study, it remains to investigate schemes relying on statistical CSIT (S-CSIT), which offers the least complex option and also incurs the lowest feedback overhead.

\section{OPTIMIZATION BASED ON S-CSIT}

Based on the channel pdf (in our case the mean $\bar{g}_{k}$ ), our objective in this section is to determine the power minimizing a fixed pair of loadings $(\mathbf{m}, \mathbf{p})$. We will consider two possibilities: in the first one we will optimize over $\mathbf{m}$ and $\mathbf{p}$, while in the second we will only optimize over $\mathbf{m}$ at uniform power loading.

\section{A. Optimum Bit and Power Loading}

Our optimization problem here is formulated as follows:

$$
\begin{aligned}
& \min _{\mathbf{m}(\overline{\mathbf{g}}) \in \mathcal{M}, \overline{\mathbf{p}}(\overline{\mathbf{g}}) \geq \mathbf{0}} J(\overline{\mathbf{p}}), \quad J(\overline{\mathbf{p}})=\sum_{k=0}^{N-1} \bar{P}_{k}(\overline{\mathbf{g}}) \\
& \text { s. to } \\
& \text { C1. } \quad \sum_{k=0}^{N-1} \frac{\log _{2} M_{k}}{R_{0}} \overline{\operatorname{BER}}_{k}\left(\bar{P}_{k}, M_{k}, \bar{g}_{k}\right)=\mathrm{BER}_{0} \\
& \text { C2. } \quad \sum_{k=0}^{N-1} \log _{2} M_{k}=R_{0} .
\end{aligned}
$$

Averaging in $\mathrm{C} 1$ needs to be carried out over all possible values of $g_{k}: \overline{\mathrm{BER}}_{k}\left(\bar{P}_{k}, M_{k}, \bar{g}_{k}\right)=\int_{0}^{\infty} e^{-\beta_{k} \bar{P}_{k} g_{k}} f_{g_{k}}\left(g_{k}\right) d g_{k}=$ $a /\left(1+\beta_{k} \bar{P}_{k} \bar{g}_{k}\right)$. Removing temporarily $\mathrm{C} 2$, as before, the KKT conditions on the relaxed problem yield

$$
\bar{P}_{k}(\overline{\mathbf{g}})=\left[\lambda_{S} \sqrt{\frac{a \log _{2} M_{k}}{R_{0} \beta_{k} \bar{g}_{k}}}-\frac{1}{\beta_{k} \bar{g}_{k}}\right]^{+}
$$

where $\lambda_{S}=\left(1 / \mathrm{BER}_{0}\right) \sum_{k=0}^{K-1} \sqrt{\left(a \log _{2} M_{k} / R_{0} \beta_{k} \bar{g}_{k}\right)}$. The sufficient conditions for optimality, $\left(\partial^{2} \bar{P} / \partial \bar{P}_{k}^{2}\right) \geq 0$ and $\left(\partial^{2} \overline{\mathrm{BER}} / \partial \bar{P}_{k}^{2}\right) \geq 0$, are easily shown to hold true, thus ensuring global optimality. As discussed earlier, (25) must be calculated for all sorted candidates $\mathbf{m}$ that meet the $R_{0}$ constraint, selecting as final solution the one which requires less power.

\section{B. Optimum Bit and Uniform Power Loading}

We finally investigate the possibility of having uniform power loading over the $N_{a}$ active subchannels. Let $\bar{P}_{u}>0$ denote the uniform power value, 1 (.) the indicator function, and define $\mathcal{P}:=\left\{\left[\bar{P}_{0} \ldots \bar{P}_{N-1}\right]^{T} \mid \bar{P}_{k}=\bar{P}_{u} \cdot \mathbf{1}\left(k \in \mathcal{N}_{a}\right)\right\}$. In this case, the search space in (24) is confined over $\mathbf{m} \in \mathcal{M}$ and $\mathbf{p} \in \mathcal{P}$. Since no analytical solution is available, we optimize as before over all feasible sorted $\mathbf{m}$ satisfying $\mathrm{C} 2$, and then numerically find $\bar{P}_{u}$ that satisfies $\mathrm{C} 1$. Finally, we choose the optimum $(\mathbf{m}, \mathbf{p})$ pair that leads to the minimum $\bar{P}_{u} N_{a}$. As $\bar{P}_{u}$ is constant across subcarriers, a simpler algorithm using a greedy approach can be implemented along the lines of [2, Ch. 16] to allocate bits, followed by a numerical search to find the value of $\bar{P}_{u}$.

\section{NUMERICAL EXAMPLES}

To numerically test our power-efficient designs, we considered an adaptive OFDM system with $N=64$ subcarriers and $B=2$ feedback bits per subcarrier (when Q-CSIT is utilized) operating over fading channels with average gains $\left\{\bar{g}_{k}\right\}_{k=0}^{N-1}$ allowed to vary over a $10 \mathrm{~dB}$ range, and prescribed to achieve $\mathrm{BER}_{0}=10^{-3}$ at rate $R_{0}=20$ bits (unless otherwise specified). 

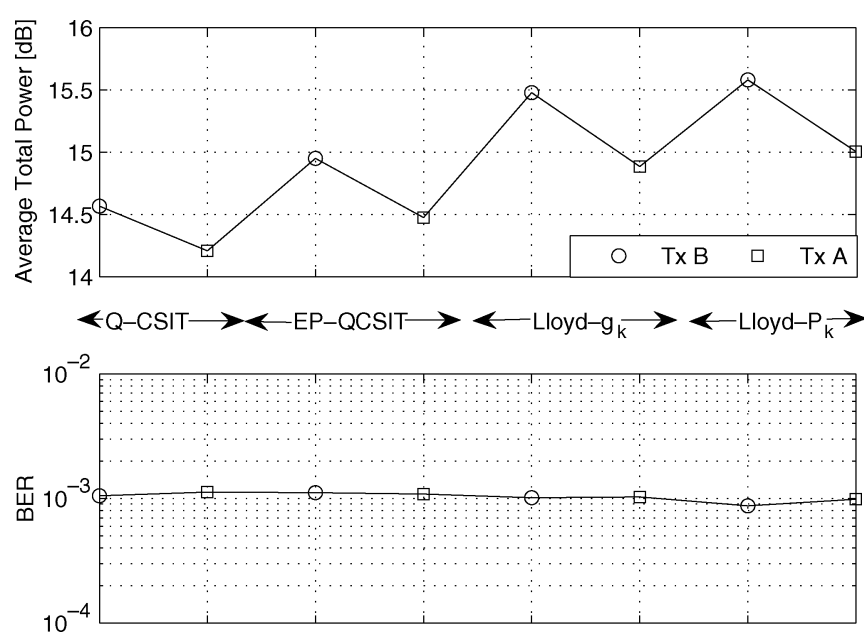

Fig. 3. Comparison of different SIT-QCSIT and Q-CSIT schemes $(N=64$, $\mathrm{BER}_{0}=10^{-3}, R_{0}=20$, and $B=2$ ).

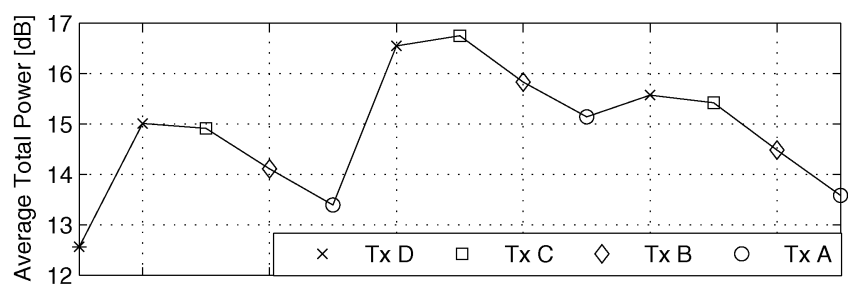

$D-C S I T \longrightarrow \longleftarrow Q C S I T \longrightarrow C T-Q C S I T \longrightarrow \longleftarrow E P-Q C S I T \rightarrow$

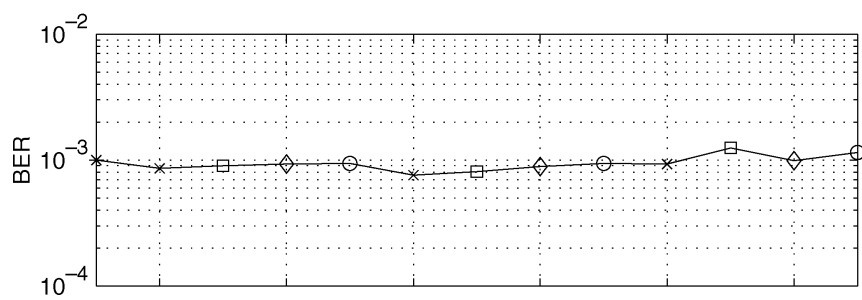

Fig. 4. Comparison of D-CSIT and Q-CSIT schemes $\left(N=64, \mathrm{BER}_{0}=\right.$ $10^{-3}, R_{0}=20$, and $B=2$ ).

Test Case 1 (SIT-QCSIT Schemes): Fig. 3 compares the optimal and reduced complexity SIT-channel quantizers; namely, EP-QCSIT, Lloyd- $g_{k}$, and Lloyd- $P_{k}$ schemes, for both Tx-A and Tx-B adaptation strategies. As expected, Tx-A always outperforms the less complex Tx-B for all schemes. Less expected is the fact that all reduced complexity quantizers suffer minimal loss (at most $1 \mathrm{~dB}$ ) in power efficiency relative to the optimum one. In particular, EP-QCSIT performs the best among suboptimum quantizers (comes to within $0.5 \mathrm{~dB}$ close to the optimum); and for this reason, we will henceforth use it as the representative of this class.

Test Case 2 (D-CSIT Versus Q-CSIT With Statistically Distinct or Equal Gains): Fig. 4 quantifies the power loss when relying on Q-CSIT rather than D-CSIT. Three schemes utilizing Q-CSIT are tested (optimum Q-CSIT, CT-QCSIT, and EP-QCSIT) for the different adaptive transmitters TxA-D. We observe that all four strategies TxA-D perform within $2 \mathrm{~dB}$ with respect to each other.
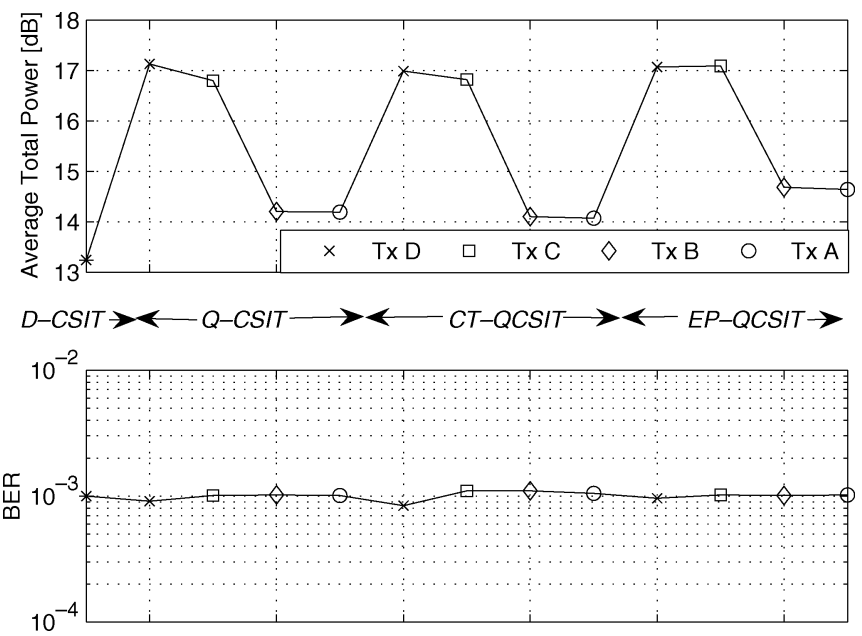

Fig. 5. Comparison of D-CSIT and Q-CSIT schemes with equal subchannels statistics $\left(N=64, \mathrm{BER}_{0}=10^{-3}, R_{0}=20, B=2\right.$, and $\left.\bar{g}_{k}=2\right)$.

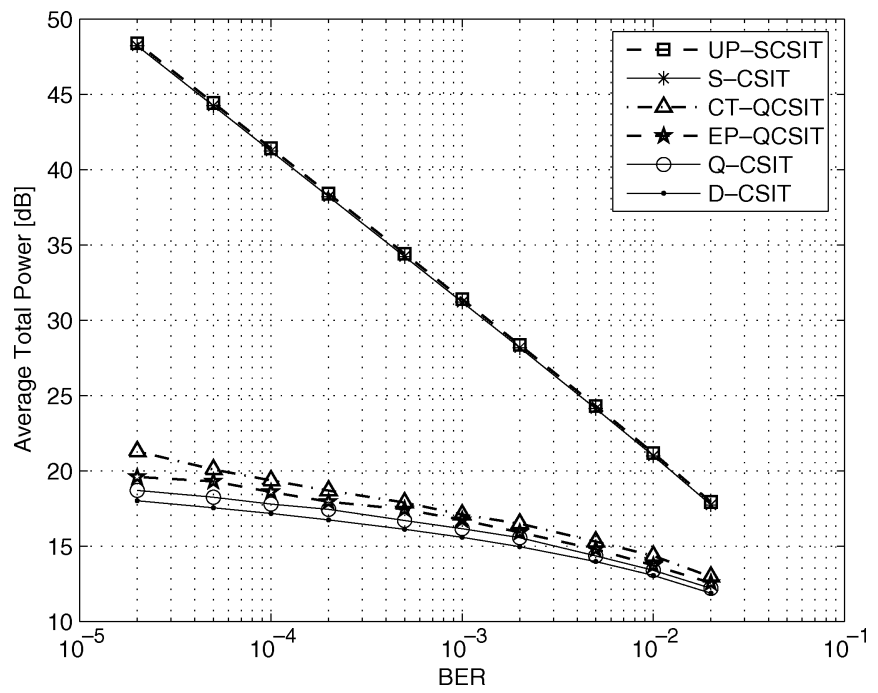

Fig. 6. Comparison of systems with different CSIT assumptions and BER constraints $\left(N=64, R_{0}=25\right.$, and $\left.B=2\right)$.

In addition, EP-QCSIT shows near-optimum power efficiency almost $1 \mathrm{~dB}$ away from the optimal D-CSIT benchmark with fully adaptive transmission (Tx-A). It is worth mentioning that the small variations in the BER curve around the $10^{-3}$ target are due to finite simulation samples.

Fig. 5 is the counterpart of Fig. 4 for $\bar{g}_{k}$ the same $\forall k$. As expected intuitively, in this case, the optimum Q-CSIT scheme exhibits performance identical with CT-QCSIT, even though the two entail different sets of thresholds. This points out the possibility that the optimum solution may not be unique. Moreover, the equivalence of Tx-A and Tx-B testifies the optimality of nearly uniform bit loading across subcarriers as the subchannels become statistically equivalent.

Test Case 3 (D-CSIT Versus Q-CSIT Versus S-CSIT in Power Efficiency and BER): For variable BER specifications, Fig. 6 includes also in the comparison optimal power loading based on S-CSIT, as well as uniform power 




Fig. 7. Comparison of D-CSIT and Q-CSIT systems with different BER constraints $\left(N=64, R_{0}=25\right.$, and $\left.B=2\right)$.

(UP) loading based on S-CSIT. We observe that the (generally nonuniformly loaded) optimum S-CSIT design is as efficient in terms of power as UP-SCSIT. Furthermore, compared with both S-CSIT-based designs, Q-CSIT exhibits an average power gain of approximately 8,15 , and $24 \mathrm{~dB}$ for $\mathrm{BER}_{0}=10^{-2}, 10^{-3}$, and $10^{-4}$, respectively. Compared with the no-CSIT case, this gain almost doubles since the S-CSIT scheme has itself a power gain in the range of $15-25 \mathrm{~dB}$. A closer look offered by Fig. 7 at both D-CSIT and Q-CSIT reveals that the optimum Q-CSIT scheme comes surprisingly close (about $1 \mathrm{~dB}$ ) to the D-CSIT scheme. In addition, the EP-QCSIT scheme comes within $1 \mathrm{~dB}$ close to the optimum Q-CSIT one. It can also be inferred that CT-QCSIT is inferior to all other schemes especially for very small BER values.

Test Case 4 (Reduced Complexity Optimization and Feedback Overhead): Here, we test suboptimal schemes with controllable complexity and feedback overhead which rely on RC1-RC4 to simplify the optimization for loading and also reduce the reverse-channel overhead by feeding back Q-CSI bits corresponding to the minimal number of subchannel gains (recall that the remaining gains are obtained via interpolation). Fig. 8 depicts the power efficiency of the various options in comparison with D-CSIT and Q-CSIT without reduced feedback overhead. Accuracy in the estimation and interpolation steps corresponding to different $\mathrm{RC}$ alternatives manifests itself in the fluctuation of the BER around its nominal value. Thanks to its simplicity and robustness to estimation errors, the RC2 option with amplitude estimation is preferable when the region is given. Notice also the negligible $0.3 \mathrm{~dB}$ loss in average total power relative to feedback-per-subchannel Q-CSIT (i.e., no feedback reduction), and the minimal $1 \mathrm{~dB}$ loss compared with the D-CSIT scheme.

Test Case 5 (Number of Quantization Regions): We finally investigate the effect of the number of feedback bits in the EP-QCSIT scheme. Fig. 9 demonstrates that power gains
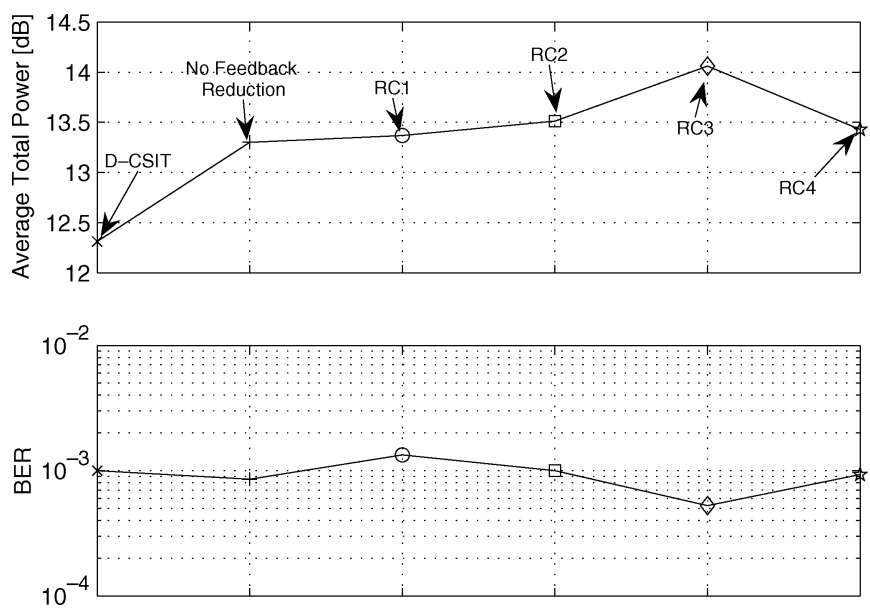

Fig. 8. Sinc interpolator with different estimation methods $\left(N=64, R_{0}=\right.$ $20, B=2, L=3, \mathbb{E}\left[\left|h_{0}\right|^{2}\right]=1, \mathbb{E}\left[\left|h_{1}\right|^{2}\right]=0.5, \mathbb{E}\left[\left|h_{2}\right|^{2}\right]=0.25$, and $\left.\mathbb{E}\left[\left|h_{3}\right|^{2}\right]=0.1\right)$.
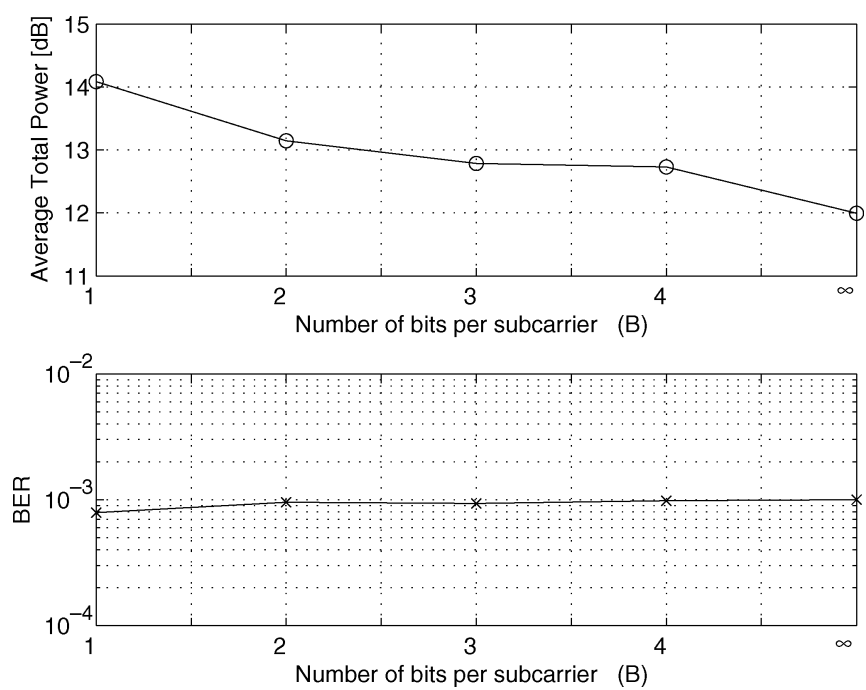

Fig. 9. Effect of number of feedback bits using the EP-QCSIT scheme ( $N=$ 64 and $R_{0}=25$ ).

increase markedly when $B$ increases from 1 to 2 bits, while a diminishing relative gain is expected for each additional increase in $B$. It also shows that having $B=1$ entails only $2 \mathrm{~dB}$ in power loss compared with the D-CSIT case which corresponds to $B=\infty$.

\section{CONCLUding Summary AND Future RESEARCH}

Under prescribed rate and error probability constraints, we optimized power-efficiency of wireless OFDM by relying on various forms of CSIT. Emphasis was placed on Q-CSIT which can become readily available because the receiver needs to feed back to the transmitter only a limited number of bits indexing the quantization region which the underlying channel falls into. The resultant optimized transceivers hold great potential since they were shown to enjoy about $15 \mathrm{~dB}$ power savings relative to S-CSIT (and twice as much when compared with OFDM with no-CSIT available), while staying within $1 \mathrm{~dB}$ from D-CSIT benchmark designs that are more suitable for slow fading wireless or wireline channels. 
To choose a judicious form of Q-CSIT, we designed offline optimal channel quantizers that rely on channel statistics (mean) to select quantization thresholds across OFDM subchannels. We further derived reduced complexity quantizers which either adopt CT for all subchannels, or, select thresholds based on individual subchannel statistics. Among the latter, the simplest one exhibiting near-optimal power savings turned out to be the one for which thresholds are selected per subchannel to render quantization regions EP. OFDM based on EP-QCSIT was seen to outperform those relying on scalar quantization based on Lloyd's algorithm, as well as those relying on CT-QCSIT.

With the designed Q-CSIT fixed, we also developed optimal and simpler suboptimal algorithms for online power and bit loading. Applying these to OFDM transmitters with variable degrees of adaptation (e.g., subcarrier or bit adaptation only) offers flexibility to tradeoff power savings for ease in implementation and reduced complexity online operation. To further simplify the operation of Q-CSIT-based designs, we relied on interpolation to devise reduced overhead feedback schemes which require Q-CSIT only on a few subchannels (as many as twice the length of the discrete-time equivalent channel). Among other comparisons, our simulations tested how the number of feedback bits affect performance and revealed that simple (EP) quantizers with 1 bit fed back per subchannel and truncated sinc interpolation can afford no more than $2 \mathrm{~dB}$ loss in power relative to the D-CSIT benchmark.

Interesting directions not explored here include the use of vector quantization for obtaining improved Q-CSIT at the price of increased complexity, as well as the possibility of feeding back a possibly different number of bits per subchannel. The promise power-efficient OFDM holds in the single-antenna point-to-point scenario considered here, motivates future research to explore its potential for power savings when multicarrier modulation is used over multiuser and (possibly distributed) multiantenna links in the context of ad hoc and wireless sensor networks.

\section{APPENDIX A \\ ON THE OPTIMALITY OF THE QUANTIZER}

While the KKT conditions are necessary, sufficient conditions for global optimality include the convexity of both objective and constraint functions [10]. Three conditions have to be satisfied to ensure convexity of $J(\overline{\mathbf{p}})=\sum_{k=0}^{N-1} \sum_{j=0}^{2^{B}-1} P_{k, j} \int_{\tau_{k, j}}^{\tau_{k, j+1}} f_{g_{k}}\left(g_{k}\right) d g_{k}$, which is a function of both $P_{k, j}$ and $\tau_{k, j}$ [10, Appendix 1]: $\left(\partial^{2} \bar{P} / \partial P_{k j}^{2}\right) \geq$ $0,\left(\partial^{2} \bar{P} / \partial \tau_{k j}^{2}\right) \geq 0$, and $\left(\partial^{2} \bar{P} / \partial P_{k j}^{2}\right)\left(\partial^{2} \bar{P} / \partial \tau_{k j}^{2}\right)-$ $\left[\left(\partial \bar{P} / \partial P_{k j} \partial \tau_{k j}\right)\right]^{2} \geq 0$. It is clear that $\left(\partial^{2} \bar{P} / \partial P_{k j}^{2}\right)=0$. It is also straightforward to show that the third condition cannot be satisfied; and hence, $J(\overline{\mathbf{p}})$ being nonconvex, the offline solution is only guaranteed to be locally optimal.

\section{APPENDIX B}

PROOF OF PROPOSITION 1

The feedback information is a quantized version of the real parameter $g_{k}$. With $\mathrm{DFT}_{N}$ denoting the DFT of length $N$ and $x[k]$ representing a sequence of length $N$ such that $x[k]=$ $\sum_{u=0}^{N-1} x_{u} \delta[k-u], g[k]$ can be expressed as

$$
\begin{aligned}
g[k] & =\frac{1}{\sigma_{k}^{2}}|H[k]|^{2}=\frac{1}{\sigma_{k}^{2}} H[k] H^{*}[k] \\
& =\frac{1}{\sigma_{k}^{2}} \operatorname{DFT}_{\mathrm{N}}\left\{h[n] \otimes h^{*}\left[((-n))_{N}\right]\right\} \\
& =\frac{1}{\sigma_{k}^{2}} \operatorname{DFT}_{\mathrm{N}}\left\{\sum_{m=0}^{N-1} h[m] h^{*}\left[((n+m))_{N}\right]\right\} \\
& \triangleq \frac{1}{\sigma_{k}^{2}} \operatorname{DFT}_{\mathrm{N}}\left\{\phi_{h h}[n]\right\}
\end{aligned}
$$

where $x^{*}$ is the conjugate of $x, \otimes$ denotes the $N$-circular convolution, and $((x))_{N}$ stands for $x$ modulo $N$. The correlation sequence $\phi_{h h}[n]$ can be shown to have the following structure (given that $N \geq 2 L+1$; otherwise, it is trivial that we revert to the feedback-per-subchannel case)

$$
\phi_{h h}[n]= \begin{cases}\sum_{m=0}^{L}|h[m]|^{2}, & n=0 \\ \sum_{m=0}^{L} h[m] h^{*}[n+m], & n=1, \ldots, L \\ \phi_{h h}^{*}[N-n], & n=N-L, \ldots, N-1 \\ 0, & \text { otherwise. }\end{cases}
$$

Using this structure of $\phi_{h h}[n]$ and (26), any gain $g_{k}$ can be expressed as

$$
\begin{aligned}
\sigma_{k}^{2} g_{k}=\phi_{h h}[0]+2 \sum_{n=1}^{L}\left[\phi_{h h, R}[n]\right. & \cos \frac{2 \pi k n}{N} \\
& \left.+\phi_{h h, I}[n] \sin \frac{2 \pi k n}{N}\right]
\end{aligned}
$$

where $\phi_{h h, R}$ and $\phi_{h h, I}$ denote, respectively, the real and imaginary parts of $\phi_{h h}$. Therefore, since we have $2 L+1$ unknowns the same number of $g_{k}$ coefficients suffices to fully describe $\phi_{h h}[n]$. This result implies that all $g_{k}$ gains can be acquired if only $2 L+1$ of them are known. As a result, $(2 L+1) B$ bits suffice to be used for feedback.

\section{REFERENCES}

[1] D. P. Bertsekas, Nonlinear Programming. Belmont, MA: Athena Scientific, 1999.

[2] T. Cormen, C. Leiserson, and R. Rivest, Introduction to Algorithms. Cambridge, MA: MIT Press, 1990.

[3] T. M. Cover and J. A. Thomas, Elements of Information Theory, ser. Telecommunications. New York: Wiley, 1991.

[4] F. F. Digham and M. S. Alouini, "Diversity combining with discrete power loading over fading channels," in Proc. Wireless Commun. Netw. Conf., Atlanta, GA, Mar. 2004, pp. 328-332.

[5] _ "Variable-rate variable-power hybrid M-FSK M-QAM for fading channels," in Proc. IEEE Veh. Technol. Conf., Orlando, FL, Oct. 2003, pp. 1512-1516.

[6] F. F. Digham and M. O. Hasna, "Performance of OFDM with M-QAM modulation and optimal loading over Rayleigh fading channels," in Proc. IEEE Veh. Technol. Conf., Los Angeles, CA, Sep. 2004, pp. 479-483.

[7] A. Gersho and R. M. Gray, Vector Quantization and Signal Compression. Norwell, MA: Kluwer, 1992.

[8] L. Goldfeld, V. Lyandres, and D. Wulich, "Minimum BER power loading for OFDM in fading channel," IEEE Trans. Commun., vol. 49, pp. 14-18, Jan. 2001 
[9] A. J. Goldsmith and S.-G. Chua, "Variable-rate variable-power M-QAM for fading channels," IEEE Trans. Commun., vol. 45, pp. 1218-1230, Oct. 1997.

[10] F. S. Hillier and G. J. Lieberman, Introduction to Operations Research. Oakland, CA: Holden-Day, Inc., 1986.

[11] D. Hughes-Hartogs, "Ensemble Modem Structure for Imperfect Transmission Media," U.S. Patents Nos 4,679,227, (Jul. 1987), 4,731,816 (Mar. 1988), and 4,833,796 (May 1989).

[12] B. S. Krongold, K. Ramchandran, and D. L. Jones, "Computationally efficient optimal power allocation algorithms for multicarrier communication systems," IEEE Commun. Lett., vol. 48, pp. 23-27, Jan, 2000.

[13] S. Lloyd, "Least squares quantization in PCM," IEEE Trans. Inf. Theory, vol. 28, pp. 129-137, Mar. 1982.

[14] D. J. Love and R. W. Heath, Jr., "Limited feedback power loading for OFDM," in Proc. Military Commun. Conf., Monterey, CA, Oct. 2004, pp. 71-77.

[15] T. K. Moon and W. C. Stirling, Mathematical Methods and Algorithms for Signal Processing. Upper Saddle River, NJ: Prentice-Hall, 2000.

[16] R. Nilsson, O. Edfors, M. Sandell, and P. O. Boerjesson, "An analysis of two-dimensional pilot-symbol assisted modulation for OFDM," in Proc. Int. Conf. Pers. Wireless Commun., Bombay, India, Dec. 1997, pp. 71-74.

[17] L. Piazzo, "Fast algorithm for power and bit allocation in OFDM systems," IEE Elect. Lett., vol. 35, pp. 2173-2174, Dec. 1999.

[18] H. Qian, C. Xiao, N. Chen, and G. T. Zhou, "Dynamic selected mapping for OFDM," in Proc. Int. Conf. Acoust., Speech, Signal Process., Philadelphia, PA, Mar. 2005, pp. 325-328.

[19] Z. Wang and G. B. Giannakis, "Wireless multicarrier communications: Where Fourier meets Shannon," IEEE Signal Process. Mag., vol. 17, no. 3, pp. 29-48, May 2000.

[20] Y. Yao and G. B. Giannakis, "Rate-maximizing power allocation in OFDM based on partial channel knowledge," IEEE Trans. Wireless Commun., vol. 4, no. 3, pp. 1073-1083, May 2005.

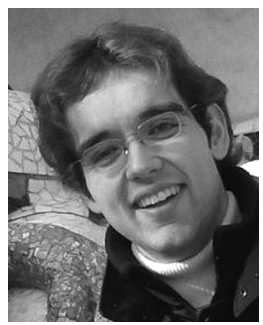

Antonio G. Marqués ( $\mathrm{S}^{\prime} 03$ ) received the Degree in Telecommunication Engineering (B.Sc. and M.Sc. degrees in electrical engineering) with Honors from the Universidad Carlos III de Madrid, Madrid, Spain, in 2002. He is currently working towards the Ph.D. degree at the Universidad Carlos III de Madrid.

In 2003, he joined the Department of Signal Theory and Communications, Universidad Rey Juan Carlos, Madrid, Spain, where he currently develops his research and teaching activities. Since 2005, he has been a Visiting Researcher at the Department of Electrical Engineering, University of Minnesota, MN. His research interests lie in the areas of communication theory, signal processing, and networking. His current research focuses on time-frequency selective channels, channel state information designs, energy-efficient resource allocation, and wireless ad hoc and sensor networks.

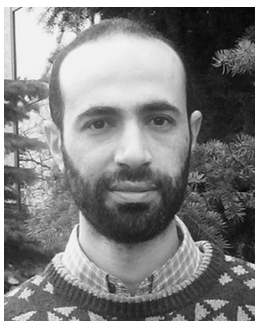

Fadel F. Digham (S'95-M'06) was born in Egypt. He received the B.Sc. degree (Hon.) and the M.Sc. degree in electronics and communications from Cairo University, Cairo, Egypt, in 1995 and 1999, respectively, and the M.E.E. and Ph.D. degrees in electrical engineering from the University of Minnesota, Minneapolis, in 2002 and 2005, respectively.

He worked for Alcatel-Telecom, Egypt branch from 1996 to 2000. He is currently a Postdoctoral Researcher with the Department of Electrical and Computer Engineering, University of Minnesota, where his current research interests are tailored to wireless communications including resource allocation, wireless sensor networks, as well as distributed signal processing and detection and estimation.

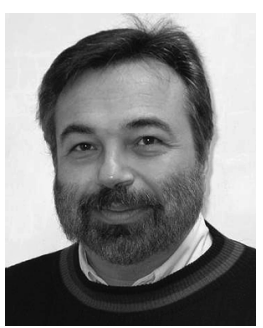

Georgios B. Giannakis (F'97) received the Diploma degree in electrical engineering from the National Technical University of Athens, Athens, Greece, in 1981. He received the M.Sc. degree in electrical engineering, the M.Sc. degree in mathematics, and the Ph.D. degree in electrical engineering, from the University of Southern California (USC), Los Angeles, CA, in 1983, 1986, and 1986, respectively.

After lecturing for one year at USC, he joined the University of Virginia in 1987, where he became a Professor of Electrical Engineering in 1997. Since 1999, he has been a Professor with the Department of Electrical and Computer Engineering, University of Minnesota, Minneapolis, where he now holds an ADC Chair in Wireless Telecommunications. His general interests span the areas of communications and signal processing, estimation and detection theory, time-series analysis, and system identification - subjects on which he has published more than 250 journal papers, 450 conference papers and four books. Current research focuses on diversity techniques for fading channels, complex-field and space-time coding, multicarrier, ultra-wide band wireless communication systems, cross-layer designs, and sensor networks.

Dr. Giannakis is the (co) recipient of six paper awards from the IEEE Signal Processing (SP) and Communications Societies (1992, 1998, 2000, 2001, 2003, and 2004). He also received Technical Achievement Awards from the SP Society in 2000 and from EURASIP in 2005. He served as Editor in Chief for the IEEE Signal Processing Letters, as Associate Editor for the IEEE TRANSACTIONS ON SIGNAL PROCESSING and the IEEE Signal Processing Letters, as Secretary of the SP Conference Board, as member of the SP Publications Board, as member and Vice-Chair of the Statistical Signal and Array Processing Technical Committee, as Chair of the SP for Communications Technical Committee, and as a member of the IEEE Fellows Election Committee. He has also served as a member of the IEEE-SP Society's Board of Governors, the Editorial Board for the Proceedings of the IEEE, and the steering committee of the IEEE TRANSACTIONS ON WIRELESS COMMUNICATIONS. 